\title{
EP-15
}

\section{Initial experience of laparoscopic liver resection in a community hospital: A case-series}

\author{
Ho-Yin LEE*, Wai-Yin CHU, Chi-Chuen MAK, Ning FAN, Ka-Wing LUI \\ Department of Surgery, Yan Chai Hospital, Hong Kong, China
}

Introduction: Laparoscopic major and anatomical hepatectomy is emerging as a preferred method to perform liver resection in recent decades. However, the role of laparoscopy remains a matter of development and has to be further evaluated. In Hong Kong, laparoscopic major and anatomical monosegmentectomy has been performing in tertiary centers with convincing outcomes. Aim of this study is to evaluate the feasibility and efficacy of laparoscopic major and anatomical liver resection to be established in a community hospital.

Methods: It is a retrospective case series between Jan 2019-Dec 2020, 20 patients underwent laparoscopic major or anatomical liver resection in Yan Chai Hospital for various indications. There are 13 cases of hepatocellular carcinoma, 6 cases of intrahepatic choledocholithiasis, 1 case of colorectal liver metastasis. hemihepatectomy was performed in 11 cases with hilar dissection; while anatomical monosegmentectomy was performed in 9 cases with Glissonean pedicle approach.

Results: There was no 30 days mortality reported in our series. 3 cases required open conversions for difficult haemostasis by laparoscopic means. The average blood loss is $723 \mathrm{~mL}+/-221 \mathrm{~mL}$ and 4 cases required intraoperative blood transfusion. The mean operative time was 325 minutes $+/-120$ minutes. There are 7 cases of post-operative collection and 1 case requiring IR-guided drainage. The average length of stay was 10.35 days.

Conclusions: Laparoscopic major and anatomical liver resection is safe and feasible in both tertiary and community center. Laparoscopic liver resection should be considered as an option whenever possible. 